\title{
Generalized Model for Memristor-Based Wien Family Oscillators
}

\author{
A. Talukdar ${ }^{1}$, A. G. Radwan ${ }^{1,2}$, and K. N. Salama ${ }^{1}$ \\ ${ }^{1}$ Electrical Engineering Program \\ King Abdullah University of Science and Technology (KAUST) \\ Thuwal, Kingdom of Saudi Arabia 23955-6900 \\ ${ }^{2}$ Department of Engineering Mathematics \\ Faculty of Engineering, Cairo University, Egypt \\ Email: \{abdul.talukdar ,ahmed.radwan,khaled.salama\}@kaust.edu.sa
}

\begin{abstract}
In this paper, we report the unconventional characteristics of memristor in Wien oscillators. Generalized mathematical models are developed to analyze four members of the Wien family using memristors. Sustained oscillation is reported for all types though oscillating resistance and time dependent poles. We have also proposed an analytical model to estimate the desired amplitude of oscillation before the oscillation starts. These memristor-based oscillation results are in good agreement with simulation results.
\end{abstract}

Keywords: memristor, Wien oscillator, non linear, oscillating resistance, dynamic poles 


\section{Introduction}

The memristor, which relates the flux-linkage $(\varphi)$ and the charge $(q)$ [1], has been the focus of enthralled research throughout the world, since HP Labs reported the first passive implementation in 2008 [2]. L. Chua first hypothesized in 1971 that the memristor achieves a resistance depending on the history of current [1]. The memristor (or memory resistor), the missing fourth basic circuit element, is a thin semiconductor film $\left(\mathrm{TiO}_{2}\right)$ sandwiched between two metal contacts. Researchers have demonstrated its revolutionary potential for use as a memory switch which offers the replacement of conventional transistor [3-7]. In recent years, the memristor has been investigated for its potential in pioneering applications such as DRAM, signal processing, neural networks, programmable logic, cross bar switch, passive switch and control systems [8-10].

Oscillators are the prime signal generators. They generate signals to be broadcasted by radio and television transmitters, as well as clock signals that regulate computers and quartz clocks [11]. Among the various types, Wien oscillator is one of the simplest forms of second order oscillators since it can generate a large range of frequencies. It has four basic structures, which constitute the Wien family [12]. The network formed with resistors and capacitors defines the condition of oscillation as well as the frequency of oscillation. Traditional Wien oscillators have the following properties: 1) all the resistors are of constant value (time independent);2) the final amplitude of the sustained oscillation can't be adjusted by circuit parameters and it depends on the nonlinearity of the op-amp, and 3) all the poles of the system will be fixed in an $s$ plane to give sustained oscillation.

In this article, we present the mathematical model for the frequency of oscillation, output amplitude and the valid frequency range for the four topologies of memristor-based Wien 
Oscillators. This study considered as electronic circuit examples of the well-known phenomena called parametric oscillator which is famous in linear time variant (LTV) system. The main aspect of these oscillators is that their state space representations have time dependent coefficients. Many examples in mechanical systems are discussed previously [13], however, the effect of time dependence came from an external source and must oscillate at one of its resonant frequencies. Here, the time dependence comes from the memristor behavior since its resistance oscillates with time as long as the output signal oscillates and with same frequencies. To validate the mathematical model, four different topologies from the same Wien family are demonstrated with their SPICE circuit simulations in this paper. These mathematical models are confirmed by simulation results which provide a clear explanation of the capability of sustained oscillations even in the presence of oscillating resistances and time-dependent oscillating poles. Same procedure can be applied for any $2^{\text {nd }}$ order memristor-based oscillator.

\section{Type 'A' Wien Oscillator with Memristor}

\subsection{Case $1: R_{1}$ is replaced by memristor}

Generally, the Wien family oscillators have the same characteristic equation, and hence the same condition of oscillation. All formulas have been generalized in fractional order sense [12], with the traditional capacitors being replaced by fractional-order ones. The concept of memristor resistance oscillation has been proved in [14] for the Wien oscillator.

In this work, we discuss the generalization of using memristors instead of resistors. The schematic of type ' $A$ ' is shown in Fig. $1 . R_{1}$ is replaced with memristor under equal capacitors, $\mathrm{C}_{1}=\mathrm{C}_{2}=\mathrm{C}=3.2 \mu \mathrm{F}$ while $\mathrm{R}_{2}=5 \mathrm{~K} \Omega$, and $\mathrm{R}_{4}=100 \mathrm{~K} \Omega$. The resistance of the memristor will be

designated as $R_{M}$ and the voltage across the memristor will be termed as $V_{M}$. Since the 
memristor resistance is time dependent, then the state space in the non-saturation conditions of this circuit can be written as follows

$$
\left(\begin{array}{l}
\frac{d V_{1}}{d t} \\
\frac{d V_{2}}{d t}
\end{array}\right)=\left(\begin{array}{cc}
-\frac{1}{R_{M}(t) C_{1}} & \frac{R_{3}}{R_{4} R_{M}(t) C_{1}} \\
-\frac{1}{R_{M}(t) C_{2}} & \frac{R_{3}}{R_{4} R_{M}(t) C_{2}}-\frac{1}{R_{2} C_{2}}
\end{array}\right)\left(\begin{array}{l}
V_{1} \\
V_{2}
\end{array}\right)=A\left(\begin{array}{l}
V_{1} \\
V_{2}
\end{array}\right)
$$

As clear from the previous differential equations, all the coefficients inside the matrix are time dependent due to the existence of memristor. In addition, the mathematical model of the memristor resistance always has the same fundamental frequency as the applied voltage as explained previously in [15- 16]. Therefore this circuit represents a good example of parametric autonomous (without excitations) oscillation in circuit design.

The stability analysis of this system can be obtained from the poles study of the characteristic equation in the s-domain which is given by

$$
s^{2}+\left(\frac{1}{R_{M}(t) C_{1}}-\frac{R_{3}}{R_{4} R_{M}(t) C_{2}}+\frac{1}{R_{2} C_{2}}\right) s+\frac{1}{R_{M}(t) R_{2} C_{1} C_{2}}=0
$$

When $R_{M}$ is constant (conventional oscillator) the necessary condition of oscillation can be obtained if trace (A) vanishes. Therefore the value of $\left(R_{3} / R_{4}\right)$ can be chosen to satisfy (3) which enforces the poles to be located on the $j \omega$ axis. While the frequency of oscillation can be given as (4)

$$
\begin{gathered}
\text { gain } \equiv \frac{R_{3}}{R_{4}}=1+\frac{R_{M}}{R_{2}} \\
f_{M}=\frac{1}{2 \pi C \sqrt{R_{M} R_{2}}}
\end{gathered}
$$

The analytical models for memristor resistance, in case of periodic input and DC waveforms, are described in $[15,16]$. These formulas are provided for both symmetrical and non-symmetrical periodic inputs. Then, as $R_{M}$ is oscillating, the swing of the amplitude of $\mathrm{R}_{\mathrm{M}}$ can be written as: 


$$
R_{\text {max }}-R_{\text {min }} \cong \frac{V_{M} k\left(R_{o f f}-R_{o n}\right)}{\pi R_{\text {init }} f_{M}}=2 \Delta R_{M}
$$

where $k=\left(\mu_{v} R_{o n} / D^{2}\right)$ as in [17], and $\mu_{v}$ is the dopant mobility. The boundaries $R_{\max }$ and $R_{\min }$, are the maximum and minimum value of $R_{M}$, i.e. $R_{M} \in\left[R_{\min }, R_{\max }\right]$. In the next sections the expression of $R_{M}$ in (5) is used to deduce our novel analytical models and to elucidate the unorthodox behaviors of memristor. Several SPICE models are proposed [17-19] to simulate the memristor as a circuit element. In this article the SPICE model, proposed in [17], is used to simulate the effect of memristor on the Wien oscillator family. The model is implemented as a SPICE sub circuit with the following parameters: the initial resistance $R_{\text {init }}$ which depends on the ratio of the doped to undoped regions at the beginning, $R_{\text {off }}=16 K \Omega, R_{\text {on }}=100 \Omega$, the width of the thin film $D=10 \mathrm{~nm}$, and the exponent $p=10$ of the window function which matches the HP-memristor [17], then only $\mathrm{R}_{\text {init }}$ is changed (from $4.1 K \Omega$ to $5.9 K \Omega$ ) in the SPICE model.

\subsubsection{Frequency of oscillation:}

As $R_{M}$ is oscillating across $R_{\text {avg }}$, the roots of the characteristic equation (2) are derived as:

$$
s=\frac{\Delta R_{M}}{2 C R_{M} R_{2}} \pm j \frac{\sqrt{4 R_{M} R_{2}-\left(\Delta R_{M}\right)^{2}}}{2 C R_{M} R_{2}}
$$

Where, $\Delta R_{M}$ is the sinusoidal amplitude as in (5).The imaginary part of $s$ gives the expression

for the frequency of oscillation. Since $R_{M}$ oscillates in the range $\left[R_{\text {avg }}-\Delta R_{M}, R_{\text {avg }}+\Delta R_{M}\right]$, then the boundaries of the oscillation frequency can be obtained as:

$$
f_{M}=\sqrt{\frac{4 R_{2}\left(R_{a v g} \pm \Delta R_{M}\right)-\left(\Delta R_{M}\right)^{2}}{16 \pi^{2} C^{2}\left(R_{\text {avg }} \pm \Delta R_{M}\right) R_{2}}}
$$


Conventional stable oscillation can only be achieved if the poles are located on the imaginary axis. Since $R_{M}$ is oscillating with time, the poles of the characteristic equation of the memristorbased oscillators become time-dependent and oscillating with time-dependent frequency as described in (7). From (5), it is observed that when $f_{M}$ increases, $\Delta R_{M}$ decreases and $R_{M}$ approaches to $R_{\text {init }}$. In Fig.2 the distance between the solid lines is the range of the memristor resistance as a function of the frequency. These solid lines show the behavior of $R_{M}$ with $f_{M}$ obtained from (5). Here we can see that for $R_{\text {init }}=5 K \Omega$, both $R_{\max }=16 K \Omega$ and $R_{\min }=100 \Omega$ tend to merge with $R_{\text {init }}$ at higher frequency. If we now plot the frequency of oscillation from (7) by replacing ( $R_{\text {avg }} \pm \Delta R_{M}$ ) with $R_{M}$ on the same graph (dotted curve in Fig. 2), two intersection points will be found. These points will give the valid frequency range where memristor can be expected to give sustained oscillation. When oscillation is sustained the system chooses the frequency where the dotted curves coincide with $R_{\text {init }}$ line which is $f_{M, a v g}$ as shown in Fig.2, at which the poles are purely imaginary. For instance, with $R_{\text {init }}=5 K \Omega, f_{M, \text { avg }}$ is found in Fig. 2 as $9.94 \mathrm{~Hz}$. From simulation $f_{M, a v g}=9.90 \mathrm{~Hz}$. This negligible difference is due to the fact that $R_{a v g}$ and $R_{\text {init }}$ differ by $0.043 \Omega$. If $R_{\text {init }}$ is increased the operational frequency range $\left(f_{2}-f_{1}\right)$ will decrease as shown in Table 1 and thus the frequency of oscillation has smaller range to settle down providing the oscillatory system has a better chance for sustained oscillation.

\subsubsection{Output voltage control:}

When simulating with SPICE the initial voltages across $C_{1}$ and $C_{2}$ set the oscillating voltage. The initial value of $C_{1}$ is set to 0.1 volt and for $C_{2}$ it is changed to see the effect on $V_{1}$ (voltage across $R_{1}$ in Fig. 1 for type 'A'). Then the initial value of $C_{1}$ is changed by keeping the initial value of $C_{2}$ at 0.1 volt. A Linear relation is found between the initial condition and $V_{1}$ for both cases which are shown in Fig. 3. Therefore, estimating the output oscillating voltage $\left(V_{\text {out }}\right)$, 
the effect of initial voltage is accounted for in Fig.3. When $R_{1}$ is replaced with $R_{M}, V_{M}$ is almost equal to $V_{1}$. Consequently $V_{M}$ can be approximated by $V_{1}$. For type 'A' Wien oscillator the relation between the output oscillation $V_{\text {out }}$ and $V_{M}$ can be derived as:

$$
\frac{V_{\text {out }}}{V_{M}}=\left(\frac{1}{1+\left(\omega R_{2} C\right)^{2}}\right)\left\{1+\frac{R_{2}}{R_{M}}+\left(\omega R_{2} C\right)^{2}-j\left(\frac{1+\omega R_{2} C^{2}+C^{2}\left(\omega R_{2}\right)^{2}}{\omega R_{M} C}\right)\right\}
$$

Here, $\omega=2 \pi f_{M}$ and $f_{M}$ can be estimated using (7). This expression can then be used to establish the amplitude of oscillation. Table 2 summarizes the comparison of the values of $f_{M}$, $V_{M}$, and $V_{\text {out }}$ for both the calculated and simulation results. In Fig.4, the errors between the simulation results and calculations are shown for both $f_{M}$ and $V_{\text {out }}$ for different $R_{\text {init }}$. We can see that as $R_{\text {init }}$ increases the percentage of error decreases. It has been established that if $R_{\text {init }}$ is increased the operational frequency range of this system (Table 1) will shorten, allowing increased possibility of sustained oscillation. So higher $R_{\text {init }}$ reduces the error for estimating $f_{M}$ and consequently, approximating $V_{\text {out }}$ using (8) gets us closer to the simulated value. Nevertheless, the nonlinear memristor behaves according to our suggested analytical expressions for any $R_{\text {init }}$ as the errors are very low for both $f_{M}$ and $V_{\text {out }}$. Previously it was not possible to calculate the amplitude of oscillation before the oscillation had begun, and to get a certain output level, another gain circuit will be added. Therefore (8) can be used to estimate the amplitude of the final oscillation which possibly helps to start the oscillation with predetermined amplitude. In Fig.5 the simulation results of the relation between output oscillation $V_{\text {out }}$ and $R_{M}$ are shown. Here the shape of the graph is elliptical. Without any external input, this elliptical shape (Fig. 5) confirms that both $V_{\text {out }}$ and $R_{M}$ are periodic. 


\subsection{Case 2: $\mathbf{R}_{2}$ as a memristor}

For this case similar simulations and analytical calculations have been followed by taking $R_{1}$ equals to $5 K \Omega$, and $R_{2}$ is replaced with memristor. The proposed formulas in the previous case are in perfect agreement with the simulation. The maximum error for $V_{\text {out }}$ is $1.5 \%$, for $R_{M}$ is $3.1 \%$, and for $f_{M}$ is $1.8 \%$. Only difference is that now there is a range of gain for which the output oscillation will be fully sustained, for instance, when $R_{\text {init }}=5 \mathrm{k} \Omega$ sustained oscillation is found for any gain in the range of $1.97-1.98$.

\section{Other Types of Wien Family Oscillator with Memristor}

In Fig. 6, the three other members of Wien oscillator are shown. In these three types either $\left(R_{1}-C_{1}\right)$ series branch or $\left(R_{2}-C_{2}\right)$ parallel branch has been interchanged with $R_{3}$ or $R_{4}$. These changes in configuration give the characteristics equation in a slightly different form, but they do not alter the frequency or conditions of oscillation; instead they share the same expressions as (3) and (4). For these three cases, $R_{1}$ is replaced with $R_{M}$ and $R_{\text {init }}$ is changed from $4.4 K \Omega$ to $5.7 K \Omega$. Surprisingly, for all types, sustained oscillations are found in spite of having the $R_{M}$ oscillating across $R_{\text {avg. }}$. From simulations it is observed that type 'C' and 'D' present similar behavior in $R_{M}$ and $V_{\text {out }}$. It may be due to the configuration of the series and parallel branch. If we look from $V_{\text {out }}$ point in Fig.6, $R_{1}-C_{1}$ series branch and $R_{2}-C_{2}$ parallel branch in type 'C' and ' $\mathrm{D}$ ' are found to be grounded, connecting by either $R_{3}$ or $R_{4}$. But type 'B' has these two branches connected in a series. Referring to Fig.7 we see that in type ' $\mathrm{B}$ ', $R_{M}$ is oscillating from $4.69 K \Omega$ to $5.2 K \Omega$ with $R_{\text {avg }}=4.945 K \Omega$ for $R_{\text {init }}=5 K \Omega$. For type 'C' and 'D' (when $\left.R_{\text {init }}=5 K \Omega\right), \mathrm{R}_{\mathrm{M}}$ is found to be oscillating from $4.842 K \Omega$ to $5.254 K \Omega$ across $R_{\text {avg }}=$ $5.048 K \Omega$. For other $R_{\text {init }}$ values similar oscillating behaviors of $R_{M}$ and $V_{\text {out }}$ are observed for 
these three types. For different $R_{\text {init }}, R_{M}$ is calculated using (5) for these three types of Wien oscillators, and the maximum errors between simulations and calculations are found to be $3.35 \%$ (for type 'B') and $0.73 \%$ ( for type 'C' and 'D'). Although $R_{M}$ is oscillating, all these Wien oscillators are found to settle down at a certain frequency when the dynamic poles of these systems are at the purely imaginary axis. Again the frequency of oscillation is calculated for each of the Wien family using (7) and the maximum error obtained for type ' $\mathrm{B}$ ' is $0.9 \%$ and for type 'C' and 'D', 1.09\%. As $R_{M}$ is oscillating for all types, poles of these systems eventually oscillate, yet make the output oscillation sustained. The gains to obtain sustained oscillations from these three types are precisely defined by $R_{a v g}$ and the purely imaginary pole. The gain does not maintain a range as mentioned in section 2.2. The gains for types 'B', 'C', and ' $\mathrm{D}$ ' are found from simulations as $1.99,2.01$, and, 2.01 respectively when $R_{\text {init }}=5 K \Omega$. When comparing these values with simulations, the maximum errors are obtained as $0.4 \%, 0.2 \%$, and $0.2 \%$

\section{Comparative Study among Memristor based Wien Family Oscillators}

In previous sections, we have summarized the Memristive effects in Wien oscillators and the analytical models also. Here we will compare the four types of memristor-based Wien oscillators (case I for type 'A'). First of all, in each type sustained oscillation is observed in spite of having the memristor's resistance $\left(R_{M}\right)$ oscillating. Fig.7 compares the simulated $R_{M}$ and $V_{\text {out }}$ for each type when $R_{\text {init }}=5 K \Omega$. In case of $R_{M}$, for type 'A' $R_{M}$ oscillates from $4.69 K \Omega$ to $5.396 K \Omega$ with $R_{\text {avg }}=5.04 K \Omega$. $R_{\text {avg }}$ gets shifted up by $0.043 K \Omega$ from $R_{\text {init }}$ for any values of $R_{\text {init }}$. For type 'B' $R_{a v g}$ is observed to shift down from $R_{\text {init }}$ by $0.055 K \Omega$. For type 'C' and ' $\mathrm{D}$ ' $R_{a v g}$ is found to shift up by $0.05 K \Omega$ for all $R_{\text {init }}$. Even with the shifting of $R_{a v g}$, oscillating $R_{M}$ results in the sustained oscillation at the output which is evident from $V_{\text {out }}$ in Fig. 7 . For other $R_{\text {init }}$ 
values similar oscillations are observed. When calculating $\mathrm{R}_{\mathrm{M}}$, a generalized model is proposed in (3) which is used to estimate $R_{M}$ for any type. The maximum errors in calculating $R_{M}$ for the four types are shown in Table 3. Note that $V_{M}$ in (5) can be approximated from $V_{1}$ which is the voltage across $R_{1}$ in the conventional circuit. In Fig. 8 the simulated results for $V_{\text {out }}, R_{M}, f_{M}$, and gain are plotted for different values of $R_{\text {init }}(4.4 K \Omega$ to $5.7 K \Omega)$. Type 'C' and ' $\mathrm{D}$ ' show exactly the same responses. All the types have a similar trend with $R_{\text {init }}$. When $R_{\text {init }}$ is increased the amplitude swing of $R_{M}$ tends to be constant, resulting in constant magnitude of output oscillation $\left(V_{\text {out }}\right)$ but with decreased frequency of oscillation $\left(f_{M}\right)$. The gain of each type needs to be increased to obtain sustained oscillation when $R_{\text {init }}$ is increased. For the purpose of calculating $f_{M}$ and gain of the circuit for any type of Wien family, generalized analytical models are suggested. The summary of the comparative results is shown in Table 3, where negligible errors affirm that these models are compatible to analyze any Wien family oscillator when memristor replaces one of the resistors. Because of oscillating $R_{M}$ one may expect $f_{M}$ to have a range, rather than to have a single value $f_{M}$ indeed has a valid operational range, as shown in Fig. 9, for all types of Wien family (when $R_{\text {init }}=5 K \Omega$ ). Type 'A' has the largest range among all types. The solid black line is obtained from (7) as all the types use (7) as generalized expressions of $f_{M}$. But $V_{M}$ varies for different types of Wien oscillator; as a result the frequency response using (5) will be different for the four types, as shown in different colors for the four types of Wien oscillator in Fig. 9. Sustained oscillation can only be obtained in that region of operation. In section 2.1.1, it is noted that if $R_{\text {init }}$ is increased this range will decrease in type 'A' but this is also true for the other three types. With the decreased operational range the error in estimating $f_{M}$ by (7) becomes less for types 'B', 'C', and 'D'. Although $f_{M}$ has a range, all the oscillatory systems choose a certain frequency at which the black solid line intersects the $R_{\text {init }}$ line. The 
chief reason for this is that when $R_{M}=R_{\text {init }}$ (more accurately $R_{\text {avg }}$ ) the pole of this second order system falls on the imaginary axis in the $s$ plane. This purely imaginary pole defines $f_{M}$ eventually. In Fig. 10, the oscillating behavior of one of the conjugate poles of Type ' $A$ ' is shown in $s$ plane when $R_{\text {init }}=5 K \Omega$. The pole will be at point $A$ when $R_{M}=R_{\max }$ and it will be at $C$ when $R_{M}=R_{\min }$. Thus the pole will move from $A$ to $C$ then $C$ to $A$ and continue oscillating for oscillating $R_{M}$. When $R_{M}=R_{\text {init }}$, that pole will be at $B$ which is on $j \omega$ axis. When the dynamic pole is at $B$, it settles the frequency of oscillation. This Perhaps could be the prime reason for the oscillatory system to have a single frequency of oscillation, bearing in mind that $R_{M}$ is oscillating. Similar behaviors of pole are also observed in types 'B', 'C', and 'D'. In order to calculate $V_{\text {out }}$ for all the types, a similar approach can be taken, as described in section 2.1.2. Because of the circuit configuration (8) will be applicable only for type 'A', but $V_{\text {out }}$ certainly can be expressed as a function of $R_{M}$ for types 'B', 'C', and ' $\mathrm{D}$ '. The method for estimating $\left.V_{\text {out }}\right)$ is shown in Fig. 11.

\section{Conclusion}

The theoretical study on the effect of using memristor in place of a resistor has been described mathematically and verified by simulation results. The behavior effect of the memristor in a second order oscillatory system are thoroughly explained with developed analytical models for gain, $R_{M}$, and $f_{M}$ to analyze the Wien family. It is now certain that sustained oscillation can be achieved despite the presence of the oscillating resistance of the memristor and time- dependent oscillating poles which is a good example for parametric oscillation. These memristor-based Wien oscillators can have greater impact in designing stable control systems, low frequency oscillators, oscillators with programmable frequency range, and high vibration-high acceleration 
systems. The same procedure can be repeated to discuss any $2^{\text {nd }}$ order Memrsitor-based oscillator to estimate the oscillating resistance, oscillating poles, gain, and the output amplitude.

\section{References}

[1] L. 0. Chua, memristor-The Missing Circuit Element, IEEE Trans. Circuit Theory, 18 (5) (1971) 507.

[2] D. B. Strukov, G. S. Snider, D. R. Stuwart, R. S. Williams, The missing memristor found, NATURE, 453 (2008) 80-83.

[3] G. Snider, Architecture and methods for computing with reconfigurable resistor crossbars, U. S. Patent 7203 789, 2007.

[4] Z. B. Yan, S. Z. Li, K. F. Wang, J. M. Liu, Unipolar resistive switching effect in YMn1- $\delta \mathrm{O}_{3}$ thin films, Appl. Phys. Lett, 96 (1) (2010) 012103

[5] H. Manem, G. S. Rose, X. He, W. Wang, Design considerations for variation tolerant multilevel CMOS/Nano memristor memory, Proceedings of the ACM Great Lakes Symposium on VLSI 2010, May 2010, pp. 287-292.

[6] F. Z. Wang, Na Helian, Wu Sinin, Mian-Guan Lim, Yike Guo, M. A. Parker, Delayed switching in memristors and memristive systems, IEEE Electron Device Letters, 31 (7) (2010) $755-757$.

[7] T. Prodromakis, K. Michelakis, C. Toumazou, Switching mechanisms in microscale memristors, Electronics Letters, 46 (1) (2010) 63-65.

[8] Y. V. Pershin, M. Di Ventra, Experimental demonstration of associative memory with memristive neural networks, Neural Networks, 23 (7) (2010) 881-886.

[9] B. L. Mouttet, Programmable crossbar signal processor, U.S. Patent 7302 513, 2007. 
[10] J. H. Young, J. Y. Lee, S. Y. Choi, J. W. Kim, Microscopic origin of bipolar resistive switching of nanoscale titanium oxide thin films, Appl. Phys. Lett, 95 (16) (2009) 162108.

[11] R. Mancini, Designing sine-wave oscillators, Edn, 45 (2000) 30-30.

[12] A. G. Radwan, A. M. Soliman, A. S. Elwakil, Design equations for fractional-order sinusoidal oscillators: four practical circuit examples, Int. J. Circuit. Theory. Applications., 36 (2008) 473-492.

[13] Komine, V., Galliou, S., and Makarov, A.: A parametric quartz crystal oscillator, Ultrasonics, Ferroelectrics and Frequency Control, IEEE Transactions on , Dec. 2003, vol.50, no.12, pp. $1656-1661$

[14] A. Talukdar, A. Radwan, K. N. Salama, Time domain oscillating poles: stability redefined in memristor based Wien-oscillator, Microelectronics (ICM), 2010 International Conference on, Dec 2010, pp. 288-291.

[15] A. G. Radwan, M. A. Zidan, K. N. Salama, On the mathematical modeling of memristors, Microelectronics (ICM), 2010 International Conference on, Dec 2010, pp. 284-287.

[16]A. G. Radwan, M. A. Zidan, K. N. Salama, HP memristor mathematical model for periodic signals and DC, Circuits and Systems (MWSCAS), $201053^{\text {rd }}$ IEEE International Midwest Symposium, Aug 2010, pp. 861-864.

[17] Z.Biolek, D. Boilek, V. Biolkova, SPICE Model of memristor with nonlinear dopant drift, Radioengineering, 18 (2) (2009) 210.

[18] M. Mahvash, M, A. C. Parker, A memristor SPICE model for designing memristor circuits, Circuits and Systems (MWSCAS), $201053^{\text {rd }}$ IEEE International Midwest Symposium, Aug 2010, pp. 989-992. 
[19] D. Batas, H. Fiedler, A memristor SPICE Implementation and a new approach for magnetic flux-controlled memristor modeling, Nanotechnology, IEEE Transactions on , 10 (2) (2011) 250255. 